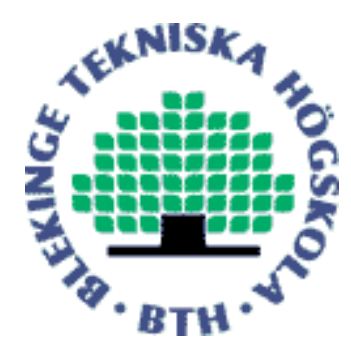

Electronic Research Archive of Blekinge Institute of Technology http://www.bth.se/fou/

This is an author produced version of a paper published in International conference on Fun and Games, Eindhoven 2008. This paper has been peer-reviewed but may not include the final publisher proof-corrections or journal pagination.

Citation for the published paper:

Lennart Nacke, Craig Lindley, Sophie Stellmach

"Log Who's Playing: Psychophysiological Game Analysis Made

Easy through Event Logging"

International conference on Fun and Games, Eindhoven 2008, The

Netherlands

DOI: 10.1007/978-3-540-88322-7_15

Access to the published version may

require subscription.

Published with permission from:

Springer 


\title{
Log Who's Playing: Psychophysiological Game Analysis Made Easy through Event Logging
}

\author{
Lennart Nacke, Craig Lindley, and Sophie Stellmach \\ Blekinge Institute of Technology, Game and Media Arts Laboratory, Box 214, \\ 37424 Karlshamn, Sweden \\ \{Lennart.Nacke, Craig.Lindley, Sophie.Stellmach\}@bth.se
}

\begin{abstract}
Modern psychophysiological game research faces the problem that for understanding the computer game experience, it needs to analyze game events with high temporal resolution and within the game context. This is the only way to achieve greater understanding of gameplay and the player experience with the use of psychophysiological instrumentation. This paper presents a solution to recording in-game events with the frequency and accuracy of psychophysiological recording systems, by sending out event byte codes through a parallel port to the psychophysiological signal acquisition hardware. Thus, psychophysiological data can immediately be correlated with in-game data. By employing this system for psychophysiological game experiments, researchers will be able to analyze gameplay in greater detail in future studies.
\end{abstract}

Keywords: psychophysiology, digital games, interactive techniques, gameplay analysis, usability.

\section{Introduction}

The automatic logging of events to better understand user behavior within an interactive system has a long history in psychology and usability [1]. Historical automated logging solutions (e.g. Skinner's "operant conditioning chamber") kept track of animal interactions (e.g. pedals pressed by a rat) to study their behavior [2]. By analyzing the response rate $\operatorname{logs}$, Skinner was able to create his theory of schedules of reinforcement. The idea of automated logging has survived until today. In recent human-computer interaction (HCI) history, behavioral observation logs are a common analysis tool. They also provide a basis for a detailed analysis of usability, fun [3] and game experience, which benefits greatly from employing classic usability metrics (e.g. time to complete a task, accuracy of input, user satisfaction) along with survey and observation measurements $[4,5]$.

Kim et al. [6] give a great overview of classic HCI instrumentations and discuss their shortcomings. They note that the amount of low-level event data recorded (e.g. number of keystrokes) is growing enormously with the complexity of modern systems. A possible solution for reducing data is to cluster them with contextual information, so that not all low-level data need to be reported. The system presented by Kim et al. combines the advantages from different research approaches, such as the 
collection of user evaluation data, qualitative survey data and behavioral data [6]. These event-related data sets can also be accompanied by video recordings to provide contextual information.

The approach of combining different methodologies to provide a coherent view of the game experience is sound. However, we find that it could be greatly improved by adding one important facet of recent game experience research: human physiological responses (as an objective context for interpreting subjective survey data). The connection of system events with human behavioral responses is gaining importance as researchers want to analyze game events with high temporal resolution [7-9].

To overcome the limitations that self-report measures of emotional responses in games have, it is of great value to assess specific game events in more detail, since some game events may trigger different or contradictory emotional physiological responses [10]. In comparison to studies investigating only tonic measures, our objective was to create a system that allows reporting of phasic psychophysiological responses at game events.

Using such a system, it is then for example possible to report emotional valence and arousal elicited by a certain game event (at a time resolution limited only by the capacities of a game engine and the psychophysiological recording hardware). In addition to eliminating the time needed for scoring game events manually using video, another key advantage of an automated logging system is its accuracy. While manual scoring might introduce errors in the log, automated scoring of game events is almost fail-safe. Integrating game events and physiological responses with eye tracker recordings and survey data can provide an almost comprehensive overview of game experience.

Because of the desire to correlate game events and psychophysiological data, this work focuses on an integrated logging framework for psychophysiological systems (like the Biosemi ActiveTwo acquisition hardware). A comprehensive framework containing various possibilities for handling different psychophysiological measurements (e.g. Biosemi ActiveTwo and Tobii 1750 eye tracker) was created as part of an internship at the BTH Game and Media Arts Laboratory [11].

- In Section 2, we introduce the design concept behind the framework. As part of the design, we investigated the requirements underlying the framework.

- Next, we present the implementation of automated event logging in detail in Section 3. This includes a look at the transmission component and the event component. It also introduces the concept of a logging entity for graphical use within a level editor.

- We end with a discussion and an outlook using our software for psychophysiological game experiments in Section 4.

\section{A Conceptual Design}

Psychophysiological researchers are interested in questions about the relationship between mind and brain or the ability to control one's well-being with your thoughts [12]. It is the curiosity about how the relation of pure feelings and thinking manifests to bodily responses that drives the research field. Andreassi defines the field as exploring the "relations between psychological manipulations and resulting physiological 
responses, measured in the living organism, to promote understanding of the relation between mental and bodily processes" [13].

\subsection{Requirements Analysis}

To gather meaningful data for further analysis, psychophysiological researchers have to record precise markers during data acquisition to manually score the recordings for further analysis, which is a serious time investment. A logging system that operates at the run-time speed of a game engine and sends out game-related events with the same temporal resolution is greatly beneficial for researchers that want to study player experience in detail. The recording of such events with the help of the same hardware that is used to record biofeedback would enable a researcher to perform an instant correlation and analysis of game experience.

In digital games one has the possibility of interacting within a virtual environment and directly influences the occurring events. For a common understanding of the types of events that occur within an experimental gaming context, we differentiate the following events:

- In-game events (e.g. player picks up a reward)

- Real-world events (e.g. shouting at the computer in anger, moving nervously on the chair)

The definition of in-game events in the source code has the advantage of logging them automatically to a file. For the examination of real-world events other methods have to be used (e.g. sensors measuring physiological responses, positional sensors, accelerometers). In our case, events need to be automatically written to a file and to the psychophysiological recording hardware via a serial cable. The semantic clustering of game events is something that deserves more thorough investigation for future studies. Nevertheless, we considered the following example events most meaningful within a first-person shooter environment (e.g. Half-Life 2):

- Player fires a gun

- Player gets hurt

- Player dies

The event must be reported to the parallel port at game runtime and simultaneously to a $\log$ file. Information in the log files needs to contain an event description and the related timestamp. The ability to log data in two different ways allows researchers without a psychophysiological recording device (that collects trigger data from a parallel port) to receive automatic output from their experiments in form of log files. In addition, the log files come in handy for debugging the functionality of the port logging.

Each event, which is reported to the parallel port, has to be transmitted for a predefined minimal time amount. If the signal is not sent long enough, older psychophysiological hardware with a lower frequency resolution might not be able to correctly register the event. Therefore, we agreed on a minimum transmission time of 200 milliseconds. 


\section{Automated Game Event Logging}

When planning the design of experimental game stimuli, we used the Source SDK that ships with the game Half-Life 2 [14, 15]. Game development tools such as the Hammer editor and access to the source code made our preference for this solution. The fact that the Source $S D K$ was used successfully in research contexts before $[16,17]$, including the development of serious games [18] also influenced our decision. Level designers should be able to easily integrate logging inside existing levels, so the logging system was designed as two modular components for Half-Life 2:

- Transmission Component

- Event Component

The transmission component is responsible for receiving signals and to communicate these to the parallel port and the log file. This is the base component on which the second module depends, because the event component will catch events anywhere in the code and report them to the transmission component.

\subsection{Transmission Component}

This module is a static $\mathrm{C}++$ class, which has to be accessible from all other files in the project to enable the possibility for firing events from any given class. For the implementation of the port logging, functionality of the InpOut32 library for communicating with the parallel port was used [19]. The basic structure for this communication shall be presented, describing the main steps as executed in the source code:

\section{Load library file \\ 2. Determine function's address \\ 3. Send event code to port \\ 4. Free library memory}

These steps are the basis for communicating with the parallel port and have been integrated within the transmission component to enable the writing of event codes to the parallel port. The process of logging to a regular text file is using an output stream. Text, represented as the datatype string or as an array of characters, is transmitted to the method $\log M s g$.

\subsection{Event Component}

The event component is responsible for managing predefined events and reports them to the transmission component. However, different kinds of events exist, which have to be handled in their certain ways. First, we have in-game events that need to be distinguished before they are reported. Some events are applicable in a general manner, whereas others are just convenient for particular scenarios. Therefore, we should differentiate between common level-independent events (e.g. player is damaged or enemy is killed) and unique level-dependent events (e.g. player enters a certain area or encounters a certain $N P C^{1}$ type).

\footnotetext{
${ }^{1}$ Non-player character: NPCs in Half-Life 2 can be either friendly or hostile.
} 


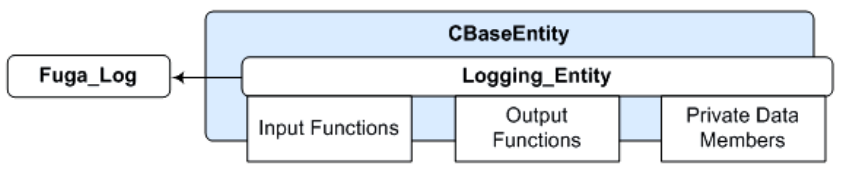

Fig. 1. Structural overview of the class logging_entity and its functions

In the specific case of Half-Life 2, the damage of a whole NPC class can be referred to as a general event. In contrast, the action by a particular $N P C$ from this class can sincerely lead to a unique event. For the design of the event component this means that the ability to dynamically assign additional unique events within the level editing tool (Hammer Editor) would be desirable. Thus, one central entity called logging_entity was created, which provides the possibility to choose from a list of common events, but also to refer to this entity by other instances for defining unique events.

Figure 1 illustrates the general structure of the logging entity. It derives from the CBaseEntity class, which is, as the name suggests, the base class for all entities contained in the Source Engine. As shown there, the members of this class can be broken down in member variables, input functions and output functions.

Available events, which can be fired by the logging entity, are indicated by its output functions. In addition, when an event is fired (not only by this but also by other entities) the mentioned input functions can be explicitly called. This means that also events by other entities can trigger the SetEventCode ${ }^{2}$ function as long as the logging entity has been given a name.

An example for calling the SetEventCode input function from another entity could be as follows. A level consists of different areas, which are sequentially playable. Every area has its distinct characteristics and it is desired to automatically log when the player moves on to a new area. A simple trigger can be created, which fires an OnTrigger output function (event), when the player passes through and sends a predefined event code to the logging class.

The work, however, is not done just by creating the class logging_entity as presented, because the output functions may be initialized, but they will not fire the desired output yet, because the exact spot in the source code, when the desired event takes place, still has to be determined. In a huge project as the Source SDK this becomes a complex and time-consuming task. But once the suitable point in the source code has been found, the approach for firing an event is always the same.

Due to the various events contained in the logging entity, working in several different source files is necessary. Thus, access to the logging entity has to be provided on a higher user level. All entities, which have been added to a certain level in the Hammer Editor, are listed in $g$ EntList. If the logging entity is not found in this list, then nothing will happen and no error will occur. Otherwise, the event defined in the logging entity will be fired and, depending on the settings of the particular level, an input function will be called.

In order to find the entity in gEntList, the logging entity has to be given a specific debug name within the Hammer Editor (e.g. "FugaLog", see Figure 2). When adding

\footnotetext{
${ }^{2}$ SetEventCode is an input function contained in logging_entity for saving a given event code to the member variable currentEventCode. With the output function SendToPort, this value will then be sent to the parallel port.
} 


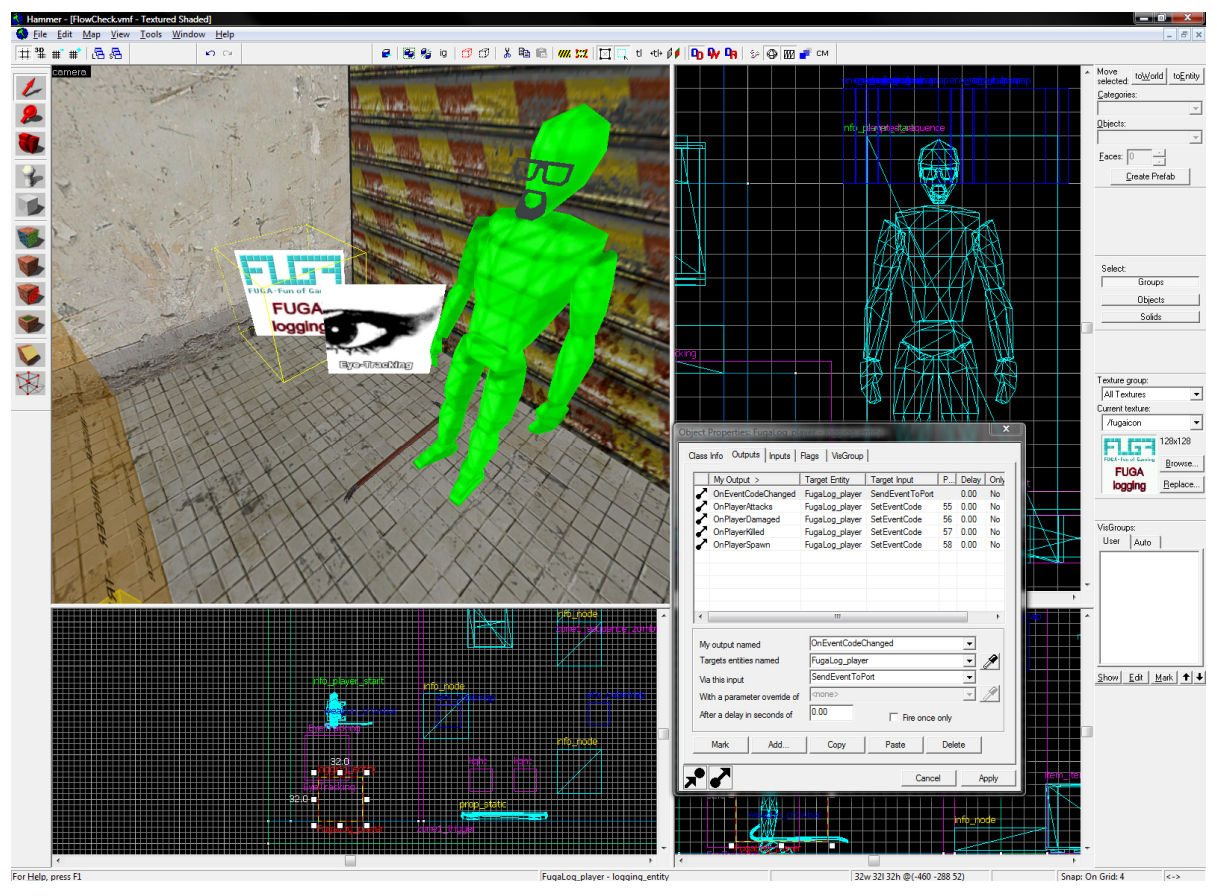

Fig. 2. Screenshot showing the logging entities and manually defined output codes inside the Hammer editing environment of the Source SDK [15]

other events the same procedure has to be followed. However, playtesting revealed that events concerning the player (e.g. "Player gets hurt") had to be distinguished from other events. Some events would, otherwise, not be logged. This had to do with the fact that closely related events would be sent simultaneously.

Designing the event component as an independent entity in the Half-Life 2 level editor (Hammer) provides several helpful features for the user:

- Existing event codes can easily be changed during level editing. The user is able to adapt event codes within a well arranged menu within the Hammer Editor. Otherwise one would have to go through thousands of lines of code in order to find the specific spot where a certain event code would be sent. Something which is impossible for ordinary users.

- It is possible to define event codes for unique events. Events closely related to a certain scenario can easily be added by defining them within the Hammer Editor. In case of the occurrence of this event, it can refer to logging_entity and send a predefined event code.

- Logging entity exceeds simple logging functionality. The logging entity can be used for more than just logging events. Output functions defined in this entity can trigger other entities. For example, if the player is damaged, a health kit should be created. This can be done by calling the function "Spawn health kit" at the event "Player is damaged". 


\section{Conclusion and Future Work}

We presented a system that writes game events to a text file and sends them simultaneously as a byte code to the port of psychophysiological recording hardware, thus making it possible to index phasic psychophysiological responses precisely at the occurrence of game events. We focused in this paper on describing the details of the implementation of this tool.

The application of the tool within empirical studies investigating game experience remains to be presented in future studies, which we have already conducted. The created logging framework presented in this study has also been used within the EU FP6 NEST FUGA project for studying the fun of gaming. Although, this paper only presents the portion of the software concerned with logging of general events, it is planned to extend the tool to visualize and cross-correlate several streams of physiological data, eye tracker data, and usability data. Thus, it will enable even more detailed analysis of player behavior.

Acknowledgments. The development of this software was supported by the European Community FP6 NEST project: The Fun of Gaming: Measuring the Human Experience of Media Enjoyment. We would like to thank Niklas Ravaja, Matias Kivikangas, Dennis Sasse, and Charlotte Sennersten for supporting us with their knowledge and valuable feedback.

\section{References}

1. Hilbert, D.M., Redmiles, D.F.: Extracting usability information from user interface events. ACM Computing Surveys 32, 384-421 (2000)

2. Skinner, B.F.: The Behavior of Organisms: An Experimental Analysis. D. AppletonCentury Company, incorporated (1938)

3. Wiberg, C.: A Measure of Fun: Extending the scope of web usability. Department of Informatics, vol. PhD. Umeå University, Umeå, Sweden (2003)

4. Nielsen, J.: Usability Engineering. Morgan Kaufmann, San Francisco (1993)

5. Bernhaupt, R., Eckschlager, M., Tscheligi, M.: Methods for evaluating games: how to measure usability and user experience in games? In: Proceedings of the international conference on Advances in computer entertainment technology, pp. 309-310 (2007)

6. Kim, J.H., Gunn, D.V., Schuh, E., Phillips, B., Pagulayan, R.J., Wixon, D.: Tracking realtime user experience (TRUE): a comprehensive instrumentation solution for complex systems. In: Proceedings of the twenty-sixth annual SIGCHI conference on Human factors in computing systems (CHI 2008). ACM, Florence, Italy (2008)

7. Ravaja, N., Turpeinen, M., Saari, T., Puttonen, S., Keltikangas-Jarvinen, L.: The Psychophysiology of James Bond: Phasic Emotional Responses to Violent Video Game Events. Emotion 8, 114-120 (2008)

8. Salminen, M., Ravaja, N.: Increased oscillatory theta activation evoked by violent digital game events. Neuroscience Letters 435, 69-72 (2008)

9. Kivikangas, J.M.: Psychophysiology of flow experience: An explorative study. Faculty of Behavioural Sciences, Department of Psychology, Vol. Master's Thesis. University of Helsinki, Helsinki, Finland (2006) 
10. Ravaja, N., Saari, T., Salminen, M., Laarni, J., Kallinen, K.: Phasic Emotional Reactions to Video Game Events: A Psychophysiological Investigation. Media Psychology 8, 343-367 (2006)

11. Stellmach, S.: A psychophysiological logging system for a digital game modification. Department of Simulation and Graphics, Bachelor thesis. Otto-von-Guericke-University, Magdeburg (2007)

12. Cacioppo, J.T., Tassinary, L.G., Berntson, G.G.: Psychophysiological science. Handbook of psychophysiology, 3-26 (2000)

13. Andreassi, J.L.: Human Behavior and Physiological Response. Psychophysiology 4, 1-12 (2000)

14. Half-Life 2 (digital game). Valve Corporation (2004)

15. Valve Developer Community (2004), http : / developer.valvesoftware. com

16. Arango, F., Chenghung, C., Esche, S.K., Chassapis, C.: A scenario for collaborative learning in virtual engineering laboratories. In: Frontiers in education conference - global engineering: knowledge without borders, opportunities without passports. FIE 2007. 37th annual (2007) F3G-7-F3G-12 (2007)

17. McQuiggan, S., Lee, S., Lester, J.: Predicting User Physiological Response for Interactive Environments: An Inductive Approach. In: Proceedings of the 2nd Artificial Intelligence for Interactive Digital Entertainment Conference, pp. 60-65 (2006)

18. Mac Namee, B., Rooney, P., Lindstrom, P., Ritchie, A., Boylan, F., Burke, G.: Serious Gordon: Using Serious Games To Teach Food Safety in the Kitchen. In: Proceedings of the 9th International Conference on Computer Games: AI, Animation, Mobile, Educational \& Serious Games (CGAMES 2006) (2006)

19. Logix4U: http:// $\log i x 4 u$.net/ 